\title{
Light Speed Invariant Solution and Its Enlightenment of Field Equation of General Relativity
}

\author{
Jian Liang Yang (iD \\ College of Physics, Zhengzhou University, Zhengzhou 450001, China \\ Correspondence should be addressed to Jian Liang Yang; bps267890@qq.com
}

Received 4 August 2020; Revised 10 September 2020; Accepted 18 September 2020; Published 29 November 2020

Academic Editor: Ghulam Abbas

Copyright ( $\odot 2020$ Jian Liang Yang. This is an open access article distributed under the Creative Commons Attribution License, which permits unrestricted use, distribution, and reproduction in any medium, provided the original work is properly cited.

\begin{abstract}
A systematic examination of the basic theory of general relativity is made, the meaning of coordinates again is emphasized, the confusion caused by unclear meaning of coordinates in the past is corrected, and the expression of the theory is made more accurate. Firstly, the equation of Einstein's gravitational field is solved in the usual coordinate system, the existence of light speed invariant solution in the spherically symmetric gravitational field is proved, and in the same time, the solution is determined. It turns out that black holes are not an inevitable prediction of general relativity. The more exact formulas for calculating the curvature of light on the surface of the Sun and the precession angle of the orbit of Mercury are given, and the convergence of general relativistic gravity and special relativistic mechanics under the weak field approximation is realized. Finally, it is shown that the coupling coefficient of the gravitational field equation is not unique. Modifying this coefficient is an ideal project to eliminate the singularities of general relativity on the condition keeping the field equation concise and elegant, and moreover, it reveals that dark matter and dark energy are the negative energy field in the matter, the expansion of the universe is the appearance of the gradual formation of galaxies in accordance with fractal rules, not only the space between galaxies is expanding but also the galaxies themselves are also expanding, new matter is continuously generated in the celestial bodies, for the first time, the unity of fractal geometry and cosmic dynamics of general relativity is realized, and the formation and evolution of galaxies are brought into the fractal generation mode. This is a living and vivacious universe in which all aspects are gradually strengthening, in sharp contrast to the dying universe under the current cosmological framework.
\end{abstract}

\section{Introduction}

Although general relativity has made some remarkable achievements, some basic problems have not been well solved, such as the physical meaning of the coordinates of Schwareschild metric, whether general relativity is the curved theory of space-time or the theory of gravity in flat space-time, whether the constant speed of light is true in the gravitational field, the singularity problem of the field equation, and whether the existence of black holes is true. The basic problem has plagued the development of general relativity [1] but also led to some confusion in practice. For example, on the one hand, the radial coordinates of Schwareschild metric are not interpreted as the normal radius, while on the other hand, the radial coordinates on the solar surface are treated as the radius of the Sun in calculating the curvature of light on the surface of the Sun, resulting in conceptual confusion. In order to deal with these problems systematically, this paper starts with the most basic problem, that is, solving the metric of the spherically symmetric gravitational field represented by coordinates in the usual sense.

\section{Spherically Symmetric Static Metric Represented in Usual Coordinates}

We just have to solve for the metric form in the usual spherical coordinates, and the form in other coordinates can be obtained by coordinate transformation. Indices $\mu, \nu, \lambda, \alpha, \beta=0,1,2,3$. Space-time coordinates $x^{\mu}=\left(x^{0}, x^{1}, x^{2}, x^{3}\right)=(t, r, \theta, \varphi)$, $x^{0}=t, x^{1}=r, x^{2}=\theta, x^{3}=\varphi$ represent the usual time, radius, and pole angles, respectively. According to the 
observational theory of general relativity, $t$ is the time recorded by a stationary observer at infinite distance, $r$ is the distance measured from the origin to another point in the gravitational field, and $\theta$ and $\varphi$ are the polar angles measured by the observer.

The discussion in this paper is carried out in the system of natural units, the speed of light of flat space-time $c=1$, and it is agreed that the space-time linear element without gravity is a flat space-time linear element:

$$
\mathrm{d} s^{2}=g_{\mu \nu} \mathrm{d} x^{\mu} \mathrm{d} x^{\nu}=\mathrm{d} t^{2}-\mathrm{d} r^{2}-r^{2}\left(\mathrm{~d} \theta^{2}+\sin ^{2} \theta \mathrm{d} \varphi^{2}\right) .
$$

According to general relativity, in a spherically symmetric gravitational field, in the coordinate system $(t, r, \theta, \varphi)$, the general form of space-time line elements is [2-7]

$$
\begin{aligned}
\mathrm{d} s^{2}= & g_{\mu \nu} \mathrm{d} x^{\mu} \mathrm{d} x^{\nu}=B(r, t) \mathrm{d} t^{2}-Q(r, t) \mathrm{d} t \mathrm{~d} r-A(r, t) \mathrm{d} r^{2} \\
& -D(r, t)\left(\mathrm{d} \theta^{2}+\sin ^{2} \theta \mathrm{d} \varphi^{2}\right) .
\end{aligned}
$$

The condition of this formula is only spherical symmetry, which is applicable to the gravitational field of both static and oscillating gravitational sources. We will just talk about static, which is what Newtonian gravity describes. The field quantity is independent of time, so the metric tensor $g_{\mu \nu}$ no longer contains time. Considering the symmetry of static time inversion, it is necessary that $g_{01}=Q(r)=0$. Therefore, for the static case of spherically symmetric, space-time line element can be written as

$$
\begin{aligned}
\mathrm{d} s^{2}= & g_{\mu \nu} \mathrm{d} x^{\mu} \mathrm{d} x^{\nu}=B(r) \mathrm{d} t^{2}-A(r) \mathrm{d} r^{2} \\
& -D(r)\left(\mathrm{d} \theta^{2}+\sin ^{2} \theta \mathrm{d} \varphi^{2}\right) .
\end{aligned}
$$

We just have to solve for three functions $B(r), A(r)$, and $D(r)$. In order to ensure that the meaning of coordinates is always clear and unchanged, this paper will not continue to simplify (3) into the so-called standard form through coordinate transformation but directly solves with the gravitational field equation. Firstly, the source external solution of the field equation, that is, the solution satisfying the vacuum field equation $R_{\mu \nu}=0$, is determined, and then the source internal solution is determined.

When the metric is expressed in ordinary coordinates, general relativity naturally becomes the theory of gravity in flat space-time, which is only higher than Newtonian gravity. But, this is the case for the observer at infinity, where space-time is flat and curved as gravity alone; for local observers, it is more like the curved theory of space-time, because the observed quantities are related to the local space-time geometry, such as the speed of time passing is different in different places. Understanding general relativity in this way does not negate the physical Riemann space, and all equations and calculation process follow the same rules, but the explanation is more delicate, easy to accurately apply general relativity.

On the other hand, from the point of view of force action, no force can accelerate a particle to more than the speed of light, and gravity is no exception. So, the limit of the speed of light should still be reflected in the gravitational field, that is, the speed limit of all motions in the gravitational field should still be 1 and the speed of light should always be 1 , relative to the observer at infinity. In this paper, we solve for this kind of metric which reflects the invariance of the speed of light, which requires $A(r)=B(r)$. For static fields that are spherically symmetric, we have

$$
\begin{aligned}
& g_{00}=B(r), \\
& g_{11}=-A(r), \\
& g_{22}=-D(r), \\
& g_{33}=-D(r) \sin ^{2} \theta, \\
& g_{\mu \nu}=0 \quad(\mu \neq \nu) .
\end{aligned}
$$

And correspondingly

$$
\begin{aligned}
& g^{00}=\frac{1}{B(r)}, \\
& g^{11}=-\frac{1}{A(r)}, \\
& g^{22}=-\frac{1}{D(r)}, \\
& g^{33}=-\frac{1}{D(r) \sin ^{2} \theta}, \\
& g^{\mu \nu}=0, \quad(\mu \neq \nu) .
\end{aligned}
$$

According to the definition $\Gamma_{\mu \nu}^{\lambda}=(1 / 2) g^{\lambda \alpha}\left[\left(\partial g_{\alpha \mu}\right.\right.$ $\left.\left./ \partial x^{\nu}\right)+\left(\partial g_{\alpha \nu} / \partial x^{\mu}\right)-\left(\partial g_{\mu \nu} / \partial x^{\alpha}\right)\right]$, repeating indices up and down means summing from 0 to 3 . Note that the connection is symmetric with respect to two subscripts and $g_{\mu \nu}$ have only diagonal terms, so when $\mu \neq \nu \neq \lambda$, we have $\Gamma_{\mu \nu}^{\lambda}=0$, and when $\mu=\nu=\lambda=a$, we have $\Gamma_{a a}^{a}=\left(g^{a a} / 2\right)\left(\partial g_{a a} / \partial x^{a}\right)$. When there are two identical indices, we have $\Gamma_{a b}^{a}=\left(g^{a a} / 2\right)\left(\partial g_{a a} / \partial x^{b}\right), \Gamma_{a a}^{b}=-\left(g^{b b} / 2\right)\left(\partial g_{a a} / \partial x^{b}\right)$, where $a$ and $b$ are specific values of $0,1,2$, and 3, so repetition does not mean summation. It is not hard to figure out all of its nonzero connections as follows [2-7]:

$$
\begin{aligned}
& \Gamma_{33}^{1}=-\frac{1}{2 A} \frac{\partial D}{\partial r} \sin ^{2} \theta, \\
& \Gamma_{23}^{3}=\frac{\cos \theta}{\sin \theta}, \\
& \Gamma_{33}^{2}=-\sin \theta \cos \theta, \\
& \Gamma_{11}^{1}=\frac{1}{2} \frac{\partial A}{\partial r}, \\
& \Gamma_{01}^{0}=\frac{1}{2 B} \frac{\partial B}{\partial r}, \\
& \Gamma_{00}^{1}=\frac{1}{2 A} \frac{\partial B}{\partial r}, \\
& \Gamma_{12}^{2}=\Gamma_{13}^{3}=\frac{1}{2 D} \frac{\partial D}{\partial r}, \\
& \Gamma_{22}^{1}=-\frac{1}{2 A} \frac{\partial D}{\partial r} .
\end{aligned}
$$


According to definition $R_{\mu \nu}=\left(\partial \Gamma_{\mu \lambda}^{\lambda} / \partial x^{\nu}\right)-\left(\partial \Gamma_{\mu \nu}^{\lambda} / \partial x^{\lambda}\right)+$ $\Gamma_{\mu \lambda}^{\alpha} \Gamma_{\alpha \nu}^{\lambda}-\Gamma_{\mu \nu}^{\alpha} \Gamma_{\alpha \lambda}^{\lambda}$, it is not hard to figure out all the components, and just to simplify things, we are going to write prime as the derivative with respect to $r$, that is, $A^{\prime}=\mathrm{d} A / \mathrm{d} r$, $A^{\prime \prime}=\mathrm{d}^{2} A / \mathrm{d} r^{2}$, and $A^{\prime 2}=(\mathrm{d} A / \mathrm{d} r)^{2}$, so we have

$$
\begin{aligned}
R_{00}= & -\frac{\partial \Gamma_{00}^{1}}{\partial r}+2 \Gamma_{01}^{0} \Gamma_{00}^{1}-\Gamma_{00}^{1}\left(\Gamma_{11}^{1}+\Gamma_{12}^{2}+\Gamma_{13}^{3}+\Gamma_{01}^{0}\right) \\
= & -\left(\frac{B^{\prime}}{2 A}\right)^{\prime}+\frac{B^{\prime 2}}{2 A B}-\frac{B^{\prime}}{2 A}\left(\frac{A^{\prime}}{2 A}+\frac{D^{\prime}}{D}+\frac{B^{\prime}}{2 B}\right), \\
R_{11}= & \frac{\partial}{\partial r}\left(\Gamma_{10}^{0}+\Gamma_{11}^{1}+\Gamma_{12}^{2}+\Gamma_{13}^{3}\right)-\frac{\partial}{\partial r} \Gamma_{11}^{1} \\
& +\left(\Gamma_{11}^{1} \Gamma_{11}^{1}+\Gamma_{12}^{2} \Gamma_{12}^{2}+\Gamma_{13}^{3} \Gamma_{13}^{3}+\Gamma_{10}^{0} \Gamma_{10}^{0}\right) \\
& -\Gamma_{11}^{1}\left(\Gamma_{11}^{1}+\Gamma_{12}^{2}+\Gamma_{13}^{3}+\Gamma_{10}^{0}\right)=\left(\frac{D^{\prime}}{D}+\frac{B^{\prime}}{2 B}\right)^{\prime} \\
& +\left(\frac{D^{2}}{2 D^{2}}+\frac{B^{\prime 2}}{4 B^{2}}\right)-\frac{A^{\prime}}{2 A}\left(\frac{D^{\prime}}{D}+\frac{B^{\prime}}{2 B}\right) .
\end{aligned}
$$$$
R_{22}=\left(D^{\prime \prime} / 2 A\right)+\left(D^{\prime} / 4 A\right)\left[-\left(A^{\prime} / A\right)+\left(B^{\prime} / B\right)\right]-1 ;
$$

$R_{33}=R_{22} \sin ^{2} \theta$. When $\mu \neq \nu$, we have $R_{\mu \nu}=0$, it means that the vacuum field equation is automatically satisfied. Therefore, we are left with the following three equations containing $B(r), A(r)$, and $D(r)$ :

$$
\begin{aligned}
-\left(\frac{B^{\prime}}{2 A}\right)^{\prime}+\frac{B^{\prime 2}}{2 A B}-\frac{B^{\prime}}{2 A}\left(\frac{A^{\prime}}{2 A}+\frac{D^{\prime}}{D}+\frac{B^{\prime}}{2 B}\right) & =0 \\
\left(\frac{D^{\prime}}{D}+\frac{B^{\prime}}{2 B}\right)^{\prime}+\left(\frac{D^{\prime 2}}{2 D^{2}}+\frac{B^{\prime 2}}{4 B^{2}}\right)-\frac{A^{\prime}}{2 A}\left(\frac{D^{\prime}}{D}+\frac{B^{\prime}}{2 B}\right) & =0 \\
\frac{D^{\prime \prime}}{2 A}+\frac{D^{\prime}}{4 A}\left(-\frac{A^{\prime}}{A}+\frac{B^{\prime}}{B}\right)-1 & =0 .
\end{aligned}
$$

From $(8) \times(1 / B)+(9) \times(1 / A)$, we obtain $-\left[\left(A B^{\prime}+\right.\right.$ $\left.\left.A^{\prime} B\right) / 2 A B\right]\left(D^{\prime} / D\right)+\left(D^{\prime} / D\right)^{\prime}+\left(D^{\prime 2} / 2 D^{2}\right)=-\left[(A B)^{\prime} / 2 A B\right]$ $\left(D^{\prime} / D\right)+\left(D^{\prime} / D\right)^{\prime}+\left(D^{\prime 2} / 2 D^{2}\right)=0$, namely, $-\left[(A B)^{\prime} /\right.$ $2 A B]+\left(D^{\prime \prime} / D^{\prime}\right)-\left(D^{\prime} / 2 D\right)=0$, integral to it we have $\ln (A B)=\int\left[\left(2 D^{\prime \prime} / D^{\prime}\right)-\left(D^{\prime} / D\right)\right] \quad \mathrm{d} r=\ln \left(D^{\prime 2} / D\right)+C_{1}$, where $C_{1}$ is the integral constant. Because $A=B=1, D=$ $r^{2}, D^{\prime}=2 r$, and $D^{\prime}=2$ at infinity, $C_{1}=-\ln 4 A B=D^{\prime 2} / 4 D$ and further we have $A^{\prime} / A=\left(2 D^{\prime \prime} / D^{\prime}\right)-\left(D^{\prime} / D\right)-\left(B^{\prime} / B\right)$; inserting it into equation (10), we get $B^{\prime}+\left(D^{\prime} / 2 D\right) B-\left(D^{\prime} / 2 D\right)=0$, which is a first-order linear ordinary differential equation with respect to $B$, and its general solution is $B=1+\left(C_{2} / \sqrt{D}\right)$, where $C_{2}$ is integral constant.

In the distance or under weak approximation, it must be that $\sqrt{D} \longrightarrow r$ and $B \longrightarrow 1-(2 G M / r)$; therefore, $C_{2}=-2 G M$, where $G$ is Newton's gravitational constant and $M$ is the mass of the source of gravitation, so we have
$B=1-(2 G M / \sqrt{D}), A=D^{\prime 2} /(4 D-8 G M \sqrt{D})$. It is important to substitute $A$ and $B$ into equation (10). We get an identity with respect to $D$, no matter what form $D=D(r)$ is, this identity can set up, and arbitrarily specifying a $D(r)$ corresponds to a unique $A(r)$ or $B(r)$, so one can pick the appropriate $D(r)$ so that $A=B$.

So far, we can say that the vacuum field equation $R_{\mu \nu}=0$ does have the solution of $A=B$, and it is unique too. This solution is not hard to solve; let us take $A=B$, then $A B=B^{2}=A^{2}=D^{\prime 2} / 4 D$, so $A=B=D^{\prime} / 2 \sqrt{D}$, namely, $1-$ $(2 G M / \sqrt{D})=B=A=D^{\prime} / 2 \sqrt{D}=[(2 \sqrt{D} d \sqrt{D}) /(2 \sqrt{D}$ $d r)]=d \sqrt{D} / d r$.

And, separating variables and integrating both sides, we get $r=\sqrt{D}+2 G M \ln (\sqrt{D}-2 G M)+C_{3}$, from which we can inversely solve for $D=D(r)$. Here, $C_{3}$ is the integral constant, which can be determined by the continuity of $D(r)$ on the surface of the gravitation source and can be ignored under the weak field approximation. In Section 5 of this paper, this integral constant will be specifically calculated.

At this point, we obtain the complete form of the spacetime line element outside the static gravitational source in the usual spherical coordinate system, which reflects the invariance of the speed of light:

$$
\mathrm{d} s^{2}=\left(1-\frac{2 G M}{\sqrt{D}}\right) \mathrm{d} t^{2}-\left(1-\frac{2 G M}{\sqrt{D}}\right) \mathrm{d} r^{2}-D\left(\mathrm{~d} \theta^{2}+\sin ^{2} \theta \mathrm{d} \varphi^{2}\right)
$$

where $\sqrt{D}=\sqrt{D}(r)$ or $D=D(r)$ meets

$$
r=\sqrt{D}+2 G M \ln (\sqrt{D}-2 G M)+C_{3} .
$$

It can be seen from (12) that, when $\sqrt{D}=2 G M$, the value of $r$ is negative infinity, while the distance of $r$ cannot be negative. Therefore, there must be $\sqrt{D}>2 G M$ and no socalled event horizon singularity, no black hole to speak of, and no interchange between space and time.

Writing (12) as $r=\sqrt{D}\left\{1+\left(C_{3} / \sqrt{D}\right)+[(2 G M \ln (\sqrt{D}-\right.$ $2 G M)) / \sqrt{D}]\}$ and using the $\operatorname{limit}_{x \rightarrow \infty}[(\ln x) / x]=0$, we know that, at infinity $r=\sqrt{D}$, in the distant or weak field approximation $r=\sqrt{D}+2 G M \ln \sqrt{D}$ or $\sqrt{D}=r-$ $2 G M \ln r$.

Finally, to emphasize that the constant speed of light is not only a theoretical requirement but also a basic observational fact, the photon that we have seen so far, no matter which star it comes from, escapes its gravity and comes to Earth at the same speed. In turn, the cosmic rays we measure, no matter which star they come from, break free of the star's gravity and reach the Earth at no more than the speed of light, even if they are very close to it. These facts are sufficient to show that light speed of 1 should still be satisfied in a gravitational field and that light moving at least in a radial direction is not affected by gravity. General relativity must fully respect this basic fact. The authors argue that not limiting the speed of light in a gravitational field is a mistake in general relativity, and it was this mistake that led to the series of mistakes that followed. 


\section{Mercury Precession and the Curvature of Light on the Sun's Surface}

Now consider the motion of Mercury around the Sun described in equation (11). Let us say that Mercury is in the plane $\theta=(\pi / 2)$ :

$$
\left(1-\frac{2 G M}{\sqrt{D}}\right)\left(\frac{\mathrm{d} t}{\mathrm{~d} s}\right)^{2}-\left(1-\frac{2 G M}{\sqrt{D}}\right)\left(\frac{\mathrm{d} r}{\mathrm{~d} s}\right)^{2}-D\left(\frac{\mathrm{d} \varphi}{\mathrm{d} s}\right)^{2}-1=0 .
$$

Geodesic $\quad\left(\mathrm{d}^{2} x^{\mu} / \mathrm{d} s^{2}\right)+\Gamma_{\alpha \lambda}^{\mu}\left(\mathrm{d} x^{\alpha} / \mathrm{d} s\right)\left(\mathrm{d} x^{\lambda} / \mathrm{d} s\right)=0$, set $\mu=0$, notice that $\mathrm{d} \theta / \mathrm{d} t=0, \Gamma_{11}^{0}=\Gamma_{12}^{0}=\Gamma_{13}^{0}=\Gamma_{00}^{0}=0$, we get

$$
\frac{\mathrm{d}^{2} t}{\mathrm{~d} s^{2}}+\Gamma_{\alpha \lambda}^{0} \frac{\mathrm{d} x^{\alpha}}{\mathrm{d} s} \frac{\mathrm{d} x^{\lambda}}{\mathrm{d} s}=\frac{\mathrm{d}^{2} t}{\mathrm{~d} s^{2}}+2 \Gamma_{10}^{0} \frac{\mathrm{d} r}{\mathrm{~d} s} \frac{\mathrm{d} t}{\mathrm{~d} s}=\frac{\mathrm{d}^{2} t}{\mathrm{~d} s^{2}}+\frac{\partial B}{\partial r} \frac{\mathrm{d} r}{\mathrm{~d} s} \frac{\mathrm{d} t}{\mathrm{~d} s} \frac{1}{B}=0 .
$$

Its solution is $\mathrm{dt} / \mathrm{d} s=a / B$, where $a$ is the integral constant. Set $\mu=3$, notice that $\mathrm{d} \theta / \mathrm{d} t=0$, $\Gamma_{11}^{3}=\Gamma_{12}^{3}=\Gamma_{00}^{3}=\Gamma_{33}^{3}=0$, we have

$$
\frac{\mathrm{d}^{2} \varphi}{\mathrm{d} s^{2}}+\Gamma_{\alpha \lambda}^{3} \frac{\mathrm{d} x^{\alpha}}{\mathrm{d} s} \frac{\mathrm{d} x^{\lambda}}{\mathrm{d} s}=\frac{\mathrm{d}^{2} \varphi}{\mathrm{d} s^{2}}+2 \Gamma_{13}^{3} \frac{\mathrm{d} r}{\mathrm{~d} s} \frac{\mathrm{d} \varphi}{\mathrm{d} s}=\frac{\mathrm{d}^{2} t}{\mathrm{~d} s^{2}}+\frac{\partial D}{\partial r} \frac{\mathrm{d} r}{\mathrm{~d} s} \frac{\mathrm{d} t}{\mathrm{~d} s} \frac{1}{D}=0 .
$$

Its solution is $\mathrm{d} \varphi / \mathrm{d} s=b / D$, where $b$ is the integral constant. Insert them to (13), use $B d r=d \sqrt{D}$, we get

$$
\frac{a^{2}}{B}-B\left(\frac{\mathrm{d} r}{\mathrm{~d} \varphi}\right)^{2} \frac{b^{2}}{D^{2}}-\frac{b^{2}}{D}-1=\frac{a^{2}}{B}-\left(\frac{\mathrm{d} \sqrt{D}}{\mathrm{~d} \varphi}\right)^{2} \frac{b^{2}}{B D^{2}}-\frac{b^{2}}{D}-1=0 .
$$

This is an ordinary differential equation with $\sqrt{D}$ as a function and $\varphi$ as an independent variable, notice that $B=1-(2 G M / \sqrt{D})$, set $\sqrt{D}=(1 / u)$, we have $d \sqrt{D}=-\left(1 / u^{2}\right) \mathrm{d} u$, and inserting it to the above equation, we gets $(\mathrm{d} u / \mathrm{d} \varphi)^{2}+u^{2}=\left(a^{2}-1 / b^{2}\right)+\left(2 G M / b^{2}\right) u+$ $2 G M u^{2}$, derivative with respect to $\varphi$ gives the orbital equation of the planet:

$$
\frac{\mathrm{d}^{2} u}{\mathrm{~d} \varphi^{2}}+u^{2}=\frac{2 G M}{b^{2}} u+3 G M u^{3} .
$$

This is an elliptic equation with precession, in the same form as the equation of the orbit of the planets that you get in your textbooks, but $u$ here is not $1 / r$ but $1 / \sqrt{D}$. The precession angle is $\delta \varphi=6 \pi(G M / r)$ when we take $r$ as the radial coordinate, where $1 / r$ is replaced by $(1 / 2)\left[\left(1 / r_{\max }\right)+\left(1 / r_{\min }\right)\right]$, where $r_{\max }$ and $r_{\min }$ stand for aphelion and perihelion respectively. So, the precession angle is now $\delta \varphi=6 \pi G M / \sqrt{D}$, where $1 / \sqrt{D}$ is replaced by $(1 / 2)\left[\left(1 / \sqrt{D_{\max }}\right)+\left(1 / \sqrt{D_{\min }}\right)\right]$ and $D_{\max }=D\left(r_{\max }\right)$ and $D_{\text {min }}=D\left(r_{\text {min }}\right)$ are the values at aphelion and perihelion, respectively, which satisfy (12). Similarly, the angle at which light bends on the surface of the Sun $\delta \varphi=4 G M_{\odot} / \sqrt{D_{e}}$, where $D_{e}=D\left(R_{\odot}\right)$ is the value at the surface of the Sun, which satisfies (12), and $R_{\odot}$ is the Sun's mass. The specific calculation can be completed after the integration constant $C_{3}$ is determined in Section 5 .
And finally, the one that needs to be emphasized is paying attention to the sign $d \sqrt{D}^{2}=(d \sqrt{D})^{2}$ and using $(1-(2 G M / \sqrt{D})) \mathrm{d} r=d \sqrt{D}$, and thus we have

$$
\mathrm{d} s^{2}=\left(1-\frac{2 G M}{\sqrt{D}}\right) d t^{2}-\left(1-\frac{2 G M}{\sqrt{D}}\right)^{-1} \mathrm{~d} \sqrt{D}^{2}-D\left(\mathrm{~d} \theta^{2}+\sin ^{2} \theta \mathrm{d} \varphi^{2}\right) .
$$

This was to be expected. Since it does not explicitly contain $r$, it can be regarded as the space-time metric with $(t, \sqrt{D}, \theta, \varphi)$ as the independent coordinate variables, and the physical content it describes is equivalent to (11) when $\sqrt{D}=\sqrt{D}(r)$ or $D=D(r)$ meets $(12)$.

\section{Link with the Mechanics of Special Relativity and the Crisis of Black Hole Fundamental}

The invariance of the speed of light described by (11) is easy to see. Suppose that the photon moves in the radial direction, $\mathrm{d} \varphi=\mathrm{d} \theta=0$ and $\mathrm{d} s^{2}=0$, and from (11), we can get $\mathrm{d} r^{2} / \mathrm{d} t^{2}=B(r) / A(r)=1$, namely, $\mathrm{d} r / \mathrm{d} t= \pm 1$, radial speed of light is constant. And now, we look at the light moving tangentially, set $\theta=\pi / 2, \mathrm{~d} r=0$, and $\mathrm{d} s^{2}=0$, the tangential speed of light is obtained from (11) by $r \mathrm{~d} \varphi / \mathrm{d} t=\sqrt{1-(2 G M / \sqrt{D})}(r / \sqrt{D})$, (12) tells us that the smaller $\sqrt{1-(2 G M / \sqrt{D})}$ is than 1 , the larger $r / \sqrt{D}$ is than 1 , so the deviation of $\sqrt{1-(2 G M / \sqrt{D})}(r / \sqrt{D})$ from 1 is actually very small, we can still think of it as 1 . It is in this sense that we say that (11) describes the speed of light invariant not strictly constant. This slight change in the tangential speed of light causes light to bend near a celestial body which otherwise travels in straight lines. The previous result is $r \mathrm{~d} \varphi / \mathrm{d} t=\sqrt{1-(2 G M / r)}$ (which can be obtained from (20)), and it is not hard to find that, when $r=2 G M$, the tangential speed of light is zero and the deviation from 1 is severe, although it means also that light can bend and does not reflect the invariance of the speed of light.

The correctness of (11) is reflected not only in the invariance of the speed of light but also in the natural connection with relativistic mechanics under the weak field approximation. (11) Provides $g_{00}=1-(2 G M / \sqrt{D})$, $g_{11}=-(1-(2 G M / \sqrt{D})), g_{22}=-D, g_{33}=-D \sin ^{2} \theta$, and $g_{\mu \nu}=0(\mu \neq \nu)$, It is easy to calculate that $\Gamma_{01}^{1}=0, \Gamma_{11}^{1}=\Gamma_{01}^{0}=$ $\{G M /[1-(2 G M / \sqrt{D})] D\} \quad \mathrm{d} \sqrt{D} / \mathrm{d} r=G M / D, \quad$ and $\Gamma_{00}^{1}=G M / D$. The dynamic equation describing the motion of free particles in a gravitational field is the geodesic equation, the proper time must be eliminated when solving for acceleration, and the geodesic equation after the elimination of proper time is $\left(\mathrm{d}^{2} x^{\mu} / \mathrm{d} t^{2}\right)+\Gamma_{\nu \lambda}^{\mu} \cdot\left(\mathrm{d} x^{\nu} / \mathrm{d} t\right) \cdot\left(\mathrm{d} x^{\lambda} / \mathrm{d} t\right)$ $-\Gamma_{\nu \lambda}^{0} \cdot\left(\mathrm{d} x^{\nu} / \mathrm{d} t\right) .\left(\mathrm{d} x^{\lambda} / \mathrm{d} t\right) .\left(\mathrm{d} x^{\mu} / \mathrm{d} t\right)=0$. It is the basic formula for calculating particle acceleration, which is derived in detail in post-Newtonian mechanics [3] and will not be repeated here.

Let the particles move in the radial direction, $d \varphi=0, d \theta=0$, set $v=\mathrm{d} r / \mathrm{d} t$, and $\mu=1$, we have

$$
\frac{\mathrm{d}^{2} r}{\mathrm{~d} t^{2}}=-\Gamma_{00}^{1}-\Gamma_{11}^{1} v^{2}+2 v^{2} \Gamma_{01}^{0}=-\frac{G M}{D}+\frac{G M v^{2}}{D}
$$


Obviously, as $v^{2}=1$, the acceleration of the particle is zero and 1 is the limit velocity. So far, we see that the invariance of the speed of light is consistent with the speed of light limit, and admitting that the speed of light is constant necessarily leads to the speed of light limit. In the distance $1 / D \longrightarrow 1 / r^{2}$, which means that, in the weak field approximation, there is $\mathrm{d}^{2} r / \mathrm{d} t^{2}=-\left(G M / r^{2}\right)+\left(G M v^{2} / r^{2}\right)$ which is exactly the mechanical equation of special relativity $\mathrm{d}(m v) / \mathrm{d} t=-\left(G M m / r^{2}\right)$, where $m=\left(m_{0} / \sqrt{1-v^{2}}\right)$ is the mass of motion of the particle. It is proved as follows:

Let a particle move in the radial direction, $v=\mathrm{d} r / \mathrm{d} t$, $\dot{v}=\mathrm{d}^{2} r / \mathrm{d} t^{2} ; \quad$ from $\mathrm{d}(m v) / \mathrm{d} t=-\left(G M m / r^{2}\right)$, we get $\mathrm{d}(m v) / \mathrm{d} t=m_{0} \mathrm{~d}\left(v / \sqrt{1-v^{2}}\right) / \mathrm{d} t=m_{0}\left\{\left[\dot{v} \sqrt{1-v^{2}}+v(1-\right.\right.$ $\left.\left.\left.v^{2}\right)^{-(1 / 2)} v \dot{v}\right] /\left(1-v^{2}\right)\right\}=m_{0} \dot{v} /\left(1-v^{2}\right)^{(3 / 2)}=-\left[G M m_{0} / r^{2}\right.$ $\left.\left(1-v^{2}\right)^{(1 / 2)}\right]$, and multiplying both sides by $m_{0} /\left(1-v^{2}\right)^{(3 / 2)}$, we get $\mathrm{d}^{2} r / \mathrm{d} t^{2}=\dot{v}=-\left(G M / r^{2}\right)+\left(G M v^{2} / r^{2}\right)$. So far, we say that (11) shows the link between the gravitational theory of general relativity with the mechanics of special relativity under the weak field approximation.

Next let us talk about the problem with the Schwareschild metric, and Schwareschild metric is

$$
\mathrm{d} s^{2}=\left(1-\frac{2 G M}{r}\right) \mathrm{d} t^{2}-\left(1-\frac{2 G M}{r}\right)^{-1} \mathrm{~d} r^{2}-r^{2}\left(\mathrm{~d} \theta^{2}+\sin ^{2} \theta \mathrm{d} \varphi^{2}\right) .
$$

Obviously (20) is also a solution that satisfies the vacuum field equation $R_{\mu \nu}=0$ in the coordinate system $(t, r, \theta, \varphi)$. Mathematically (20) and (11) are equal weight but describe different physical processes. It is worth saying because of existing singularity $r=2 G M$, (20) must be defective. Although the curvature of the Sun's rays and the precession of Mercury calculated with it are consistent with observations, it does not mean that it is entirely correct, because it causes serious problems in other ways. For example, it can lead to faster-than-light motion in flat time space, directly contradicting the basis of special relativity.

In order to avoid the obvious defect existing in (20), the ordinary textbooks do not interpret the $r$ there as the usual radius, but instead refer to it as the radial parameter with fuzzy meaning or standard coordinates or something else $[1-8]$. But, this is unhelpful and leads only to conceptual confusion, for since the curvature of the Sun's surface and the precession angle of Mercury have been calculated with $r$ as the usual radius, there should be no other explanation and the basic concept must not be ambiguous. Obviously, the $r$ in (20) cannot be associated with the perihelion of Mercury's orbit or the radius of the Sun if it does not give it the meaning of usual radius.

The predicted black hole is also the result of treating the $r$ in (20) as the usual radius. Here is how a black hole is predicted: let the light move along the radial direction, $d \theta=d \varphi=0, \quad d s^{2}=0 ; \quad$ from (20), we get $\mathrm{d} r / \mathrm{d} t= \pm[1-(2 G M / r)]$; on the surface, $r=2 G M$, we have $\mathrm{d} r / \mathrm{d} t=0$. The result is that, from the observer's point of view at infinity, the photon stops on the surface, the matter inside the surface cannot emit light, and the light from outside the surface can never reach the surface. That is where black holes come from. Obviously, if we do not give $r$ the meaning of usual radius, normal vector diameter, $(\mathrm{d} r / \mathrm{d} t)$ does not have the meaning of normal velocity. We should realize that the space-time metric is not a linear superposition of the metric of each independent celestial body, so the black hole must be formed by a relatively isolated system. If there are other substances with nonnegligible mass around, the symmetry of the sphere is bound to be destroyed, and (20) will lose the premise of existence. In this case, the metric needs to be resolved from the field equation.

Next, it is shown how (20) predicts faster-than-light motion in flat space-time. (20) Provides $g_{00}=1-(2 G M / r)$, $g_{22}=-r^{2}, \quad g_{11}=-[1-(2 G M / r)]^{-1}, \quad g_{33}=-r^{2} \sin ^{2} \theta$, and $g_{\mu \nu}=0(\mu \neq \nu)$. It is easy to calculate that $\Gamma_{01}^{0}=G M /$ $\left(r^{2}-2 G M r\right), \quad \Gamma_{01}^{1}=0, \quad \Gamma_{11}^{1}=-G M /\left(r^{2}-2 G M r\right), \quad$ and $\Gamma_{00}^{1}=(1-2 G M / r) G M / r^{2}$. Insert them into $\mathrm{d}^{2} x^{\mu} /$ $\mathrm{d} t^{2}+\Gamma_{\nu \lambda}^{\mu} \mathrm{d} x^{\nu} / \mathrm{d} t . \mathrm{d} x^{\lambda} / \mathrm{d} t-\Gamma_{\nu \lambda}^{0} \mathrm{~d} x^{\nu} / \mathrm{d} t . \mathrm{d} x^{\lambda} / \mathrm{d} t . \mathrm{d} x^{\mu} / \mathrm{d} t=0$, and let the particle moves in the radial direction, $d \varphi=d \theta=0$, $\nu=\mathrm{d} r / \mathrm{d} t$, and set $\mu=1$, we have

$$
\frac{\mathrm{d}^{2} r}{\mathrm{~d} t^{2}}=-\Gamma_{00}^{1}-\Gamma_{11}^{1} v^{2}+2 v^{2} \Gamma_{01}^{0}=-\left(1-\frac{2 G M}{r}\right) \frac{G M}{r^{2}}+\frac{3 G M v^{2}}{\left(r^{2}-2 G M r\right)}
$$

Clearly as $v^{2}>1 / 3, \mathrm{~d}^{2} r / \mathrm{d} t^{2}=-[1-(2 G M / r)]\left(G M / r^{2}\right)+$ $\left\{3 G M v^{2} /[1-(2 G M / r)] r^{2}\right\}>-\left(G M / r^{2}\right)+\left\{3 G M v^{2} /[1-\right.$ $\left.(2 G M / r)] r^{2}\right\}>\left(3 v^{2}-1\right)\left(G M / r^{2}\right)$, and we can see that the direction of the acceleration is actually going outward. We know that electromagnetic forces can accelerate charged particles to near the speed of light 1 , so we can expect a large enough accelerator or other device at $r_{0}$ of the gravitational field to accelerate a charged particle to $v_{0}>1 / \sqrt{3}$ and then let it shoot out in the radial direction, and we want to know if it is traveling at infinity faster than the speed of light.

For this sake, let us calculate the velocity of the particle at infinity described by the equation $\mathrm{d}^{2} r / \mathrm{d} t^{2}=$ $\left(3 v^{2}-1\right)\left(G M / r^{2}\right)$, and the actual velocity is obviously greater than that. Since $\mathrm{d}^{2} r / \mathrm{d} t^{2}=v d v / d r=\mathrm{d} v^{2} /$ $2 \mathrm{~d} r=\left(3 v^{2}-1\right)\left(G M / r^{2}\right)$, the solution is $1-3 v^{2}=$ $k \exp (-6 G M / r)$, where $k$ is the integral constant. For $r \longrightarrow \infty, 1-3 v_{\infty}^{2}=k$, when the particle travels outward from $r_{0}$ with initial velocity $v_{0}$, the velocity at infinity is

$$
v_{\infty}=\sqrt{\frac{1}{3}-\left(\frac{1}{3}-v_{0}^{2}\right) \exp \frac{6 G M}{r_{0}}} .
$$

It can be seen that, when $v_{0}>\sqrt{1 / 3}$, as long as $\left(6 G M / r_{0}\right)$ are large enough, the velocity of the particle reaching the infinite distance can be greater than 1 , which is not allowed by the basic assumption of special relativity. It shows that (20) is not suitable to describe the strong gravitational field and high-speed motion, so the black hole predicted by it is not reliable. If the electromagnetic force in the gravitational field cannot make the initial velocity of a charged particle $v_{0}>\sqrt{1 / 3}$, this violates the independence of the electromagnetic force and gravity; there is no reason to think that the electromagnetic equipment in the gravitational field cannot make the velocity of a charged particle close to the speed of light 1 . 
Since 2015, detectors have been claiming to detect gravitational waves from black holes, but there has been intense opposition [8-14], with opponents arguing that LIGO detects nothing more than noise. The authors argue that black holes do not probably exist and that even gravitational waves detected do not come from them, deliberately avoiding the meaning of coordinates, treating general relativity as a mathematical game out of reality, and not developing relativity but burying it. At the other extreme is to abandon general relativity, build other models of the universe, and explain phenomena sporadically, all of which are very superficial.

\section{Invariant Solution of the Speed of Light in a Spherically Symmetric Static Gravity Source}

First of all, when we say that the speed of light in the gravitational source does not change, we do not mean that the speed of light moving in the medium does not change but that the speed of light moving through a hole in the gravitational source does not change, that is, it is still equal to 1 . That means that $A(r)=B(r)$ still holds in the source. From the following solution's process, it can be seen that such a solution not only exists but also is unique.

The equation of the gravitational field is $R_{\mu \nu}=\gamma\left(T_{\mu \nu}-(1 / 2) T g_{\mu \nu}\right)$, and its another form is $R_{\mu \nu}-(1 / 2) R g_{\mu \nu}=\gamma T_{\mu \nu}$, where $\gamma$ is the coupling constant and $T_{\mu \nu}=(\rho+p) u_{\mu} u_{v}-p g_{\mu \nu}$ is the energy-momentum tensor of the gravitational source. From definition $u^{\mu}=\mathrm{d} x^{\mu} / \mathrm{d} s, u_{\mu}=g_{\mu \nu} u^{\nu}, \quad u_{\mu} u^{\mu}=1, \quad g_{\mu \nu} g^{\mu \nu}=4, \quad$ and $T=g^{\mu \nu} T_{\mu \nu}=\rho-3 p$. For the static source $u^{i}=\mathrm{d} x^{i} / \mathrm{d} s=0$, $i=1,2,3$. From (3), we know $g_{00}=B(r), g_{11}=-A(r), g_{22}=-D(r), g_{33}=-D(r) \sin ^{2} \theta$, and $g_{\mu \nu}=0(\mu \neq \nu)$, then $u_{i}=g_{i v} u^{\nu}=0, \quad i=1,2,3$. So, $1=B(\mathrm{~d} t / \mathrm{d} s)^{2}=B\left(u^{0}\right)^{2}, \quad u_{0}=g_{00} u^{0}=\sqrt{B}$, $T_{11}=p A, T_{22}=p D, T_{33}=p D \sin ^{2} \theta$, and $T_{00}=\rho B$, and now the equations (8)-(10) can be changed as

$$
\begin{gathered}
R_{00}=-\left(\frac{B^{\prime}}{2 A}\right),+\frac{B^{\prime 2}}{2 A B}-\frac{B^{\prime}}{2 A}\left(\frac{A^{\prime}}{2 A}+\frac{D^{\prime}}{D}+\frac{B^{\prime}}{2 B}\right) \\
=\gamma\left(T_{00}-\frac{T}{2} g_{00}\right)=\frac{\gamma}{2}(\rho+3 p) B, \\
R_{11}=\left(\frac{D^{\prime}}{D}+\frac{B^{\prime}}{2 B}\right)^{\prime}+\left(\frac{D^{\prime 2}}{2 D^{2}}+\frac{B^{\prime 2}}{4 B^{2}}\right)-\frac{A^{\prime}}{2 A}\left(\frac{D^{\prime}}{D}+\frac{B^{\prime}}{2 B}\right) \\
=\gamma\left(T_{11}-\frac{T}{2} g_{11}\right)=\frac{\gamma}{2}(\rho-p) A, \\
R_{22}=\frac{D^{\prime \prime}}{2 A}+\frac{D^{\prime}}{4 A}\left(-\frac{A^{\prime}}{A}+\frac{B^{\prime}}{B}\right)-1=\gamma\left(T_{22}-\frac{T}{2} g_{22}\right) \\
=\frac{\gamma}{2}(\rho-p) D .
\end{gathered}
$$

From $\left(R_{00} / 2 B\right)+\left(R_{11} / 2 A\right)+\left(R_{22} / D\right)$, we see $(1 / A)^{\prime}+$ $\left[\left(2 D^{\prime \prime} / D^{\prime}\right)-\left(D^{\prime} / 2 D\right)\right] \quad(1 / A)-\left(2 / D^{\prime}\right)-2 \gamma \rho\left(D / D^{\prime}\right)=0$, which is a first-order linear ordinary differential equation with respect to $(1 / A)$, and its general solution is

$$
\begin{gathered}
\frac{1}{A}=e^{-\int\left(\left(2 D^{\prime \prime} / D^{\prime}\right)-\left(D^{\prime} / 2 D\right)\right) \mathrm{d} r} \int\left(\frac{2}{D^{\prime}}+2 \gamma \frac{\rho D}{D^{\prime}}\right) \\
e^{\int\left(\left(2 D^{\prime \prime} / D^{\prime}\right)-\left(D^{\prime} / 2 D\right)\right) \mathrm{d} r} \mathrm{~d} r \\
=\frac{\sqrt{D}}{D^{\prime 2}}\left(4 \sqrt{D}+4 \gamma \int \rho D d \sqrt{D}\right) \\
\text { or } A=\frac{D^{\prime 2}}{4 D\left(1+\sqrt{D}^{-1} \gamma \int \rho D d \sqrt{D}\right)} .
\end{gathered}
$$

Because of the weak field approximation $D \longrightarrow r^{2}, A$ has to be close to 1 , so the definite solution is

$$
\begin{aligned}
A & =\frac{D^{\prime 2}}{4 D\left(1+\sqrt{D}^{-1} \gamma \int_{0}^{\sqrt{D}} \rho D \mathrm{~d} \sqrt{D}\right)} \\
& =\frac{1}{\left(1+\sqrt{D}^{-1} \gamma \int_{0}^{\sqrt{D}} \rho D \mathrm{~d} \sqrt{D}\right)}\left(\frac{\mathrm{d} \sqrt{D}}{\mathrm{~d} r}\right)^{2}=A_{1}{\sqrt{D^{\prime}}}^{2},
\end{aligned}
$$

where $A_{1}=1 /\left(1+\sqrt{D}^{-1} \gamma \int_{0}^{\sqrt{D}} \rho D \mathrm{~d} \sqrt{D}\right)$, pay attention to the signs $\sqrt{D}^{\prime}=\mathrm{d} \sqrt{D} / \mathrm{d} r, \sqrt{D}^{\prime 2}=(\mathrm{d} \sqrt{D} / \mathrm{d} r)^{2}, \sqrt{D}^{\prime \prime}=$ $\mathrm{d}^{2} \sqrt{D} / \mathrm{d} r^{2}$, and insert these into (25) and notice that $A^{\prime}=A_{1}^{\prime} \sqrt{D}^{\prime 2}+2 A_{1} \sqrt{D}^{\prime} \sqrt{D}^{\prime \prime}, \quad D^{\prime \prime}=(\sqrt{D} \cdot \sqrt{D})^{\prime \prime}=$ $2 \sqrt{D}^{\prime 2}+2 \sqrt{D} \sqrt{D^{\prime \prime}}, \quad B^{\prime}=\mathrm{d} B / \mathrm{d} r=(\mathrm{d} B / \mathrm{d} \sqrt{D})(\mathrm{d} \sqrt{D} / \mathrm{d} r) ;$ we have

$$
\frac{\mathrm{d} B}{B \mathrm{~d} \sqrt{D}}=-\gamma A_{1}\left(p \sqrt{D}+D^{-1} \int_{0}^{\sqrt{D}} D \rho \mathrm{d} \sqrt{D}\right) .
$$

whose general solution is $B=\exp \int\left[-\gamma A_{1}\left(p \sqrt{D}+D^{-1} \int_{0}^{\sqrt{D}} D \rho \mathrm{d} \sqrt{D}\right)\right] \mathrm{d} \sqrt{D}$, and to make $B(r)$ continuous at the boundary, the definite solution is

$$
\begin{aligned}
& B=\left(1-\frac{2 G M}{\sqrt{D_{e}}}\right) \\
& \quad \exp \int_{\sqrt{D_{e}}}^{\sqrt{D}}\left[-\gamma A_{1}\left(p \sqrt{D}+D^{-1} \int_{0}^{\sqrt{D}} D \rho \mathrm{d} \sqrt{D}\right)\right] \mathrm{d} \sqrt{D},
\end{aligned}
$$

where $D_{e}=D\left(r_{e}\right)$ is the value of $D(r)$ at the boundary and $r_{e}$ is the radius of the source. Notice that $r$ still does not appear in (28) or (29) in the explicit form. Similar to the external solution, substituting (27) and (29) into any of the equations (23)-(25), we get the identity respect with to $D$, that is, this is true regardless of the function of $D=D(r)$, regardless of the value of $\gamma$. So, we can pick some function $D=D(r)$ such that $A=B$ is determined by the following equation: 


$$
\begin{aligned}
& \left(1-\frac{2 G M}{\sqrt{D_{e}}}\right) \exp \int_{\sqrt{D_{e}}}^{\sqrt{D}}\left[-\gamma A_{1}\left(p \sqrt{D}+D^{-1} \int_{0}^{\sqrt{D}} D \rho \mathrm{d} \sqrt{D}\right)\right] \\
& \mathrm{d} \sqrt{D}=\frac{\sqrt{D}^{\prime 2}}{\left(1+\sqrt{D}^{-1} \gamma \int_{0}^{\sqrt{D}} \rho D \mathrm{~d} \sqrt{D}\right)} .
\end{aligned}
$$

On the other hand, we know $T_{\mu ; \nu}^{v}=0$ from the field equation, that is, the covariant divergence of the gravitational source is zero, also known as the conservation law, which describes the motion state of the continuum within the gravitational source (geodesics describe the free motion of a single particle). For a static source, this equation becomes $[2-7]$

$$
\frac{\partial p}{\partial x^{\mu}}=-(\rho+p) \frac{\partial}{\partial x^{\mu}} \ln \sqrt{B} .
$$

\section{Modification of the Coupling Constant of Gravitational Field Equation and the Source Internal Solution of $D(r)$}

When the pressure at the surface of the gravitational source is set as zero, it can be seen from (28) that if the geodesic equation is required to return to Newtonian gravity under the weak field approximation, the coupling constant $\gamma$ must be equal to $-8 \pi G$, which is the previous result. But, this result should be considered wrong, because it leads to a lot of singularities that should not occur. One of the most common singularities is that when the ratio of the mass to the radius of an object is $(2 G M / R)>(8 / 9)$, the pressure inside the object becomes infinite [3], which is obviously absurd [15]. Pressure can never be allowed to be infinite, it means that the celestial bodies cannot exist, and $2 G M / R$ is in principle arbitrary, which at least should not be restricted by general relativity. Pressure appears infinite which shows our theory is not perfect yet, and this is the reason why this paper tries to modify the coupling constant. As we will see, when the pressure is negative, the coupling constant is identified as $4 \pi G$, which not only eliminates singularities but also solves cosmological problems.

I need to say a few words about negative pressure. In the history of physics, there have been countless times when negative quantities have been squeezed out, but ultimately they have proved to hinder scientific progress. It is reasonable to believe that rejecting negative pressure is also a bias.

Einstein's original explanation of this pressure term showed that he did not refuse to take a negative value. In the book, "the meaning of relativity," the Princeton University Press Published (1922) (page 117), for $T_{\mu \nu}=\rho u_{\mu} u_{\nu}$, Einstein said "we shall add a pressure term that may be physically established as follows. Matter consists of electrically charged particles. On the basis of Maxwell's theory, these cannot be conceived of as electromagnetic fields free from singularities. In order to be consistent with the facts, it is necessary to introduce energy terms, not contained in Maxwell's theory, so that the single electric particles may hold together in spite of the mutual repulsion between their elements, charged with electricity of one sign. For the sake of consistency with this fact, Poincare has assumed a pressure to exist inside these particles which balances the electrostatic repulsion. It cannot, however, be asserted that this pressure vanishes outside the particles. We shall be consistent with this circumstance if, in our phenomenological presentation, we add a pressure term. This must not, however, be confused with a hydrodynamical pressure, as it serves only for the energetic presentation of the dynamical relations inside matter." From this statement, it can be seen that Einstein did not equate pressure as a source of gravity with the dynamic pressure of a fluid but regarded it as a phenomenological representation of all the action within matter, including the electromagnetic force. It is not surprising that a negative value is taken.

Now let us solve for $D(r)$ in the source with negative pressure and determine the coupling constant. For the convenience of calculation, we solve the $D(r)$ in a spherically symmetric gravity source with uniform density $\rho=$ const. We should understand density is a statistical concept, the density of a point refers to the average value of a small range near the point, so for some not too big celestial bodies such as the Sun, that their density is regarded as constant is reasonable, which is equivalent to the whole body as a statistical volume element, so the $D(r)$ solved here are suitable for the sun and other objects that are not too big.

When $\rho$ is regarded as a constant, $p=-\rho$ is obviously the solution to (31). Since the geodesic under the weak field approximation must return to Newtonian gravity, there is $\Gamma_{00}^{1} \longrightarrow\left(G M / r_{e}^{2}\right)$ on the surface of the source, and it is not difficult to see from (28) the coupling constant $\gamma=4 \pi G$.

Notice that $\Gamma_{00}^{1}=-\left(1 / 2 g_{11}\right) \mathrm{d} g_{00} / \mathrm{d} r=(1 / 2 B)$ $(\mathrm{d} B / \mathrm{d} \sqrt{D})(\mathrm{d} \sqrt{D} / \mathrm{d} r), \quad p=-\rho=-\left(3 M / 4 \pi r_{e}^{3}\right)$, under the weak field approximation $\sqrt{D} \longrightarrow r, \quad \mathrm{~d} \sqrt{D} / \mathrm{d} r \longrightarrow 1$, $A_{1} \longrightarrow 1, \quad 4 \pi \int_{0}^{\sqrt{D_{e}}} \rho D \mathrm{~d} \sqrt{D} \longrightarrow 4 \pi \int_{0}^{r_{e}} \rho r^{2} \mathrm{~d} r=M$. Take $p=-\rho$ and $D_{e}$ as constants and $\gamma=4 \pi G$, the integral of both sides of (30) is easy to work out, and we get

$$
\frac{\mathrm{d} \sqrt{D}}{\mathrm{~d} r}=\sqrt{\frac{1-\left(2 G M / \sqrt{D}_{e}\right)}{1+\left(4 \pi G \rho D_{e} / 3\right)}}\left(1+\frac{4 \pi G \rho D}{3}\right) .
$$

The general solution of the equation is obtained by the method of separation of variables $\sqrt{3 / 4 \pi G \rho}$ $\operatorname{arctg} \sqrt{4 \pi G \rho D / 3}=r \sqrt{\left(1-2 G M / \sqrt{D}_{e}\right) /\left(1+4 \pi G \rho D_{e} / 3\right)}+$ $C_{4}$. As $r=0$ and $B^{\prime}=0$, which is because the force on the particle at the origin is zero, use (28), at the origin $D=D(0)=0$; as a result, $C_{4}=0$; then, the definite solution of (32) is

$$
\sqrt{\frac{3}{4 \pi G \rho}} \operatorname{arctg} \sqrt{(4 \pi G \rho D / 3)}=r \sqrt{\frac{1-(2 G M / \sqrt{D} e)}{1+\left(4 \pi G \rho D_{e} / 3\right)}} .
$$

This is the relationship between $D$ and $r$ in the gravitational source which meets the requirement of constant speed of light and is an implicit function. By the way, the 
reason why the field equation can accommodate additional conditions is ultimately that the metric tensor satisfies Bianchi identity, the ten components of the field equation are not completely independent, and the additional conditions can be added before the equation can be solved.

Apply (33) to the surface of the source and set $D=D_{e}=D\left(r_{e}\right)$, we can solve $D_{e}$ on the boundary. In the specific calculation, we still need to make an approximate treatment. We expand both sides of (33) by Taylor and take a second-order approximation; notice that $\rho=3 M / 4 \pi r_{e}^{3}$ and plug in $D_{e} / r_{e}^{3}=1 / r_{e}$ are taken as first-order approximation, we have

$$
\begin{aligned}
& \sqrt{D_{e}}-\frac{G M D_{e}}{9 r_{e}^{3}}=\sqrt{D_{e}}-\frac{G M}{9 r_{e}}=r_{e} \sqrt{\frac{1-\left(2 G M / \sqrt{D}_{e}\right)}{1+\left(4 \pi G \rho D_{e} / 3\right)}} \\
& =r_{e}\left(1-\frac{3 G M}{2 r_{e}}\right) .
\end{aligned}
$$

So, $\sqrt{D_{e}}=r_{e}-(7 / 6) G M$ at the boundary.

So far, we can determine the integral constant $C_{3}$ in (12). Apply (12) to the gravitational source, i.e., the surface of the celestial body, and set $\sqrt{D_{e}}+2 G M \ln \left(\sqrt{D_{e}}-\right.$ $2 G M)+C_{3}=r_{e}$ and make an appropriate approximation, we have

$$
\begin{aligned}
C_{3} & =r_{e}-\sqrt{D_{e}}-2 G M \ln \left(\sqrt{D_{e}}-2 G M\right) \\
& =r_{e}-\sqrt{D_{e}}-2 G M \ln r_{e}=\frac{7 G M}{6}-2 G M \ln r_{e} .
\end{aligned}
$$

With the determination of $C_{3}$, the calculations of Mercury precession angle and light bending can be completed. The calculated results are little different from the original ones and conform to the observations.

Next, it needs to be proved that the modification of the inverse square force of $D(r)$ obtained on the surface of the celestial body is within the allowable range of observation, that is, it has no contradiction with observation. Taking the sun as an example, it can be seen from (19) that the gravitational acceleration at the surface of the sun $g \equiv \Gamma_{00}^{1}=-(G M / D)$. Notice that the boundary is also where $\sqrt{D}$ deviates the most from $r$, and the further we go, the smaller will be the deviation. So, we just have to look at the correction of the inverse square force at the boundary.

For the sun, its $r_{e}=R_{\odot}=6.96 \times 10^{8} \mathrm{~m}$ and $M=M_{\odot}=1.989 \times 10^{30} \mathrm{~kg}$, so on its surface, $\sqrt{D_{e}}=R_{\odot}-$ $(7 / 6) G M_{\odot}$ and $D_{e} \approx R_{\odot}^{2}-(7 / 3) R_{\odot} G M_{\odot}=R_{\odot}^{2}\left[1-\left(7 G M_{\odot}\right.\right.$ $\left.\left./ 3 R_{\odot}\right)\right]$; then, the acceleration of gravity at the surface is $g=$ $-\left(G M_{\odot} / D_{e}\right)=-\left(G M_{\odot} / R_{\odot}^{2}\right)-\left(7 G M_{\odot} / 3 \quad R_{\odot}\right)\left(G M_{\odot} / R_{\odot}^{2}\right)$ $=-\left(G M_{\odot} / R_{\odot}^{2}\right)-4.9 \times 10^{-6}\left(G M_{\odot} / R_{\odot}^{2}\right)$. It can be seen that the gravitational acceleration on the surface of the sun increases by 4.9 parts per million of the inverse square force, which is negligible and consistent with observations. This means that the $D(r)$ obtained here is reasonable. Notice that $G M_{\odot} / R_{\odot}=2 \times 10^{-6}$ is a dimensionless number, and in IU, it is $G M_{\odot} / c^{2} R_{\odot}$.
It should also be noted that (20) also implies a correction of the inverse square force, which gives the acceleration of gravity at the surface of the sun:

$$
g=-\Gamma_{00}^{1}=-\frac{G M_{\odot}}{R_{\odot}^{2}}\left(1-\frac{2 G M_{\odot}}{R_{\odot}^{2}}\right)=-\frac{G M_{\odot}}{R_{\odot}^{2}}+4.5 \times 10^{-6} \frac{G M_{\odot}}{R_{\odot}^{2}} .
$$

It requires the inverse square force to be reduced by 4.5 parts per million, and the deviation is small, which is why (20) is a good description of low-speed motion.

In a word, the coupling constant of field equation can be modified as $4 \pi G$, the field equation is now written as $R_{\mu \nu}-(1 / 2) R g_{\mu \nu}=4 \pi G T_{\mu \nu}$, and correspondingly, the pressure $p$ takes negative.

A more detailed discussion of this modified coupling coefficient in Cartesian coordinates is available in [16].

\section{Further Interpretation of the Physical Significance of the Negative Pressure}

Einstein did not interpret the pressure term in a gravitational source as the dynamic pressure of a fluid but as a phenomenological representation of the pressure within a matter to balance the electromagnetic force and prevent charged particles from being disintegrated by electrical repulsion, which we should accept. Einstein did not point out that from what power the pressure is produced; today, it is easy to infer as follows: to prevent the disintegration of the protons and neutrons is the strong force, and to prevent the disintegration of electronics is the weak force and the electromagnetic force. Therefore, the pressure term should be understood as a phenomenological representation of the combined effects of the strong, weak, electromagnetic, gravitational, and all other forms of action within a matter, representing the total binding energy that holds the matter together, represented by the potential energy of the system. In other words, if you divide the matter infinitely and move each part to infinity, the work done is the volume integral of the pressure, which is negative, and the absolute value is equal to the mass of the gravitational source, namely,

$$
\int p \mathrm{~d} x \mathrm{~d} y \mathrm{~d} z=-M
$$

which is like adding a physical condition to make the solution of the pressure definite. In fact, the form of the gravitational source already determines the interpretation of $p$. As gravitational source $T_{\mu \nu}=(\rho+p) u_{\mu} u_{\nu}-p g_{\mu \nu}$, if $p$ is still understood as the normal dynamic pressure, then the field equation can only be used to solve for the metric of an ideal fluid, which is obviously not required by general relativity and the dynamic pressure in a solid is generally considered to be zero; obviously, $p=0$ does not satisfy (31). Can $p$ be understood as a thermal pressure? No, because the thermal pressure is absorbed by $\rho$ in the form of thermal kinetic energy and cannot be repeated to appear.

When the field equation with the coupling constant $4 \pi G$ is applied to the universe, $T_{\mu \nu}=(\rho+p) u_{\mu} u_{\nu}-p g_{\mu \nu}$ 
represents the energy-momentum tensor of space of the universe, and taking the statistical average of $\rho$ and $p$, we have $p=-\rho$, which is just the equation of state of the dark energy said usually. Therefore, we say that the dark energy is just the binding energy of matter, rather than an objective existence independent of matter. It turns out that the mysterious dark energy we are looking for day and night is the binding energy of matter, and the concept of dark energy will be a thing of the past.

\section{Fractal Generation Model of Galaxy Formation}

Cosmic space is treated as isotropic, and the metric that describes this kind of space-time in a co-move coordinate system $(t, l, \theta, \varphi)$ is Robson-Walker metric:

$$
\mathrm{d} s^{2}=\mathrm{d} t^{2}-R^{2}(t)\left[\frac{1}{1-k l^{2}} \mathrm{~d} l^{2}+l^{2} \mathrm{~d} \theta^{2}+l^{2} \sin ^{2} \theta \mathrm{d} \varphi^{2}\right],
$$

where $R(t)$ is the cosmic scale factor, $k$ is a constant, and $l$ is the radial coordinate, in other books, it is denoted as $r$, just to distinguish it from the usual radius, here $l$ instead of $r$. When the field equation $R_{\mu \nu}-(1 / 2) R g_{\mu \nu}=4 \pi G T_{\mu \nu}$ with coupling constant $4 \pi G$ is applied to the universe, that is, combined with Robson-Walker metric, the following two equations are obtained:

$$
\begin{aligned}
& \left(\frac{\mathrm{d} R}{\mathrm{~d} t}\right)^{2}+k=-\frac{4 \pi G}{3} \rho R^{2}, \\
& \frac{\mathrm{d} \rho}{\mathrm{d} t} R+3 \frac{\mathrm{d} R}{\mathrm{~d} t}(p+\rho)=0 .
\end{aligned}
$$

Equation (39) shows that $k$ is negative, which proves that space-time is infinite, that is, open. (39) is similar to the original Friedman equation; just replace the $G$ there with - (G/2) to get (39). (40) is the so-called energy equation, which is in the same form as the original result. Now, put $p=-\rho$ in (40), get $p=-\rho=$ const, which means that the density and pressure remain the same as the universe expands or contracts, and new matter must be created continuously in the universe.

Notice that the density is constant, and from the derivative of both sides of (39), it is not hard to find a general solution to (38):

$$
R(t)=k_{1} \sin \left(t \sqrt{\frac{4 \pi G \rho}{3}}+k_{2}\right) .
$$

It shows that the universe expands and contracts in cycles, with two integral constants $k_{1}$ and $k_{2}$. Considering that time has no beginning and no end, the moment of $R(t)=0$ has occurred countless times. Let us define the nearest moment of $R(t)=0$ as zero, which is the moment $t=0$, then $k_{2}=0$. It is easy to calculate that $\mathrm{d} R / \mathrm{d} t=k_{1} \sqrt{4 \pi G \rho / 3} \cos (t \sqrt{4 \pi G \rho / 3})$ and Hubble parameter $H(t) \equiv \mathrm{d} R / \mathrm{Rd} t=\sqrt{4 \pi G \rho / 3} \cos$ $(t \sqrt{4 \pi G \rho / 3})$.
Since everything disappears at $R(t)=0[16]$, including light, the universe in the last cycle is unobservable and no concern to us. What we care about is the age of our universe, which is the time from the beginning $(t=0)$ of the most recent cycle to today, and using $H(t)=$ $\sqrt{4 \pi G \rho / 3} \cos (t \sqrt{4 \pi G \rho / 3})$, we obtain our universe's age:

$$
t_{0}=\frac{1}{\sqrt{(4 \pi G \rho / 3)}} \arctan \sqrt{\frac{4 \pi G \rho}{3 H_{0}^{2}}} .
$$

By substituting the observed density $\rho=3.1 \times 10^{-28} \mathrm{~kg} / \mathrm{m}^{3}$ of the universe and the Hubble parameter of today $H_{0}=H\left(t_{0}\right)=70 \mathrm{~km} \cdot \mathrm{s}^{-1} \cdot \mathrm{Mpc}^{-1}$ into the above equation, we get $t_{0}=1.37 \times 10^{10}$ years, i.e., 13.7 billion years, which is the same as the previous theoretical results.

It can be seen from (41) that the cyclical period of expansion and contraction of the universe is $2 \pi / \omega=\sqrt{3 \pi / G \rho}=$ $2 \times 10^{11}$ years, namely, 200 billion years, so the universe is currently in the expansion stage and will begin to contract after rest 36.3 billion years. Contraction is the reverse course of expansion.

The new field equation gives the new relation between distance and reshift of cosmology:

$$
H_{0} d_{L}=\frac{z+1}{\sqrt{q_{0}+1}} \ln \frac{(z+1) \sqrt{q_{0}+1}+\sqrt{\left(q_{0}+1\right)(z+1)^{2}-q_{0}}}{1+\sqrt{q_{0}+1}},
$$

which fits well with the observational data of distance and reshift, and the detailed derivation of the relation can be seen in [16]. And again, show that the universe's expansion is at a decelerated rate, and with the emergence of negative pressure, the introduction of dark matter and dark energy becomes redundant. Here, $d_{L}$ is the luminosity distance and $q_{0}$ is the deceleration parameter today.

On the other hand, since the negative pressure is confined to the inner part of the celestial body, the formation of new matter can only be generated in the celestial body. Apply the field equation to a celestial body, that is, from $T_{\nu ; \mu}^{\mu}=0$, get $\mathrm{d} m=\mathrm{d}(\rho V)=-p \mathrm{~d} V=\rho \mathrm{d} V$, whose more detailed derivation can be found in [16]. Here, $V$ is the volume of the object, $m$ is its mass, and $p$ and $\rho$ are its internal pressure and density, the average of both is taken. Of course, the result is also suitable to apply to a galaxy.

In order to keep the density of the universe unchanged during the expansion process, the galaxy or celestial body must grow with the expansion of the universe and satisfy $V \propto R^{3}(t)$. Since $\mathrm{d} m=\rho \mathrm{d} V=(m / V) \mathrm{d} V$, the integral is $m=C V, C$ is integral constant, so $m \propto R^{3}(t)$. In other words, for any two moments $t_{1}$ and $t_{2}$, the mass of a galaxy or celestial body meets $m\left(t_{1}\right) / m\left(t_{2}\right)=R^{3}\left(t_{1}\right) / R^{3}\left(t_{2}\right)$.

This gives a picture of the evolution of the universe: everything is expanding, not only the space between galaxies is expanding, but the galaxies themselves are expanding, and new matter is continuously being created within them. Since the whole and the part follow the same generation law- Hubble expansion-the universe has an infinite nested self-similar fractal structure, the formation process of the 
galaxy is the generation process of the fractal, the whole universe is an infinite and constantly growing fractal, and Hubble expansion is the iterative relationship.

Many people have recognized the fractal structure of the universe, but they are unwilling to explain the formation of galaxies by the gradual growth mechanism of fractal formation $[12-14,16-19]$. The biggest obstacle is that they cannot explain the formation of new matter. Now, with $\mathrm{d} m=\rho \mathrm{d} V$, the formation of matter is no longer a problem.

Previous theories of galaxy formation believed that galaxies were formed by the gradual accumulation of matter that existed after the Big Bang, which in effect acknowledged that galaxies that existed long ago evolved only in their form and thus could not explain the fractal structure of galaxies. Observations and experiments tell us that all fractals are formed from nothing and grow as a whole. For example, the growth of organisms, the accumulation of electrodes, and the formation of lightning are all specific fractal generation processes. However, unlike the growth of organisms, the growth of galaxies is not formed by absorbing environmental material, there is no material exchange between galaxies, and the galaxies are growing simultaneously, so the increase of galactic material can only be achieved through their own internal action; $p$ and $\rho$ represent both sides of the unity of opposites.

Figure 1 is a step-by-step magnification of the solar system. It represents the actual growth process of the solar system. With the universe expanding, the solar system is also becoming bigger and bigger, not only size and mass increase but also brightness increases. For example, the radius of the Earth is expanding today at a speed of $v=H_{0} r_{0}=0.5(\mathrm{~mm} /$ year $)$, where $r_{0}=6400 \mathrm{~km}$ is the radius of today's Earth. Correspondingly, the Earth's mass increases at a rate of $3 H_{0} m_{0}=1.2$ (billion tons/year), where $m_{0}=5.96 \times 10^{24} \mathrm{~kg}$ is the Earth's mass today, and in the same time, the Earth is moving away from the sun at a velocity of $v=H_{0} d_{0}=9(\mathrm{~m} /$ year $)$; here, $d_{0}=1.49 \times 10^{8} \mathrm{~km}$ is the distance between the sun and the Earth today.

Figure 2 is a step-by-step magnification of the Milky Way. It represents the actual growth process of the Milky Way. As the universe expands, not only its size and mass increase but also its brightness increases. Of course, in the inverse process galaxies gradually disappears, corresponding to the contraction course of the universe [16]. For example, the radius of the galactic disk is expanding at a rate of $v=H_{0} d_{0}=900(\mathrm{~m} / \mathrm{s})$, and $d_{0}=7000$ light years is the disk radius of the Milky Way today.

Figure 3 is a step-by-step magnification of a piece of cosmic space, which represents the actual expansion process of cosmic space. The white spot in the image represents galaxies; not only the space between galaxies is expanding but also the galaxies themselves are expanding. It shows that the universe is an infinite fractal, with a constant density as it grows, just as organisms do. Therefore, we conclude that, in the course of universal expansion, celestial bodies are getting bigger, stars are getting brighter, and the planets that do not shine will evolve into the stars that do. The evolution of celestial brightness and temperature can be seen in [16].
We should be aware that all kinds of conservation laws summarized by people at present (including conservation of energy and conservation of mass) are the refinement of phenomena and processes occurring in a small scope within a short time, and these conservation laws should be modified accordingly once they are extended to a large scope or a large time. That is to say, the symmetry in space can only be approximately true over a small range, and as the range of observation expands, the symmetry must break and the break will become more and more obvious. Similarly, the symmetry in time, namely, the conservation, will gradually appear a bit of nonconservation with the increase of the time span, until the conservation cannot be seen completely. In fact, anything and any process can be considered unchanged as long as the time of observation is short enough. Cicadas only know summer and autumn but do not know winter and spring because of their short life span. Compared with the evolution of the universe, human's life span is similar to that of cicadas. So, their understanding of things is also limited and partial. The only thing people can do is to consciously and actively overcome this illusion caused by too short observation's time.

For a more detailed discussion of the expansion process of the universe and the fractal structure of galaxies, see the author's paper and related papers [17-23]. It should be pointed out that in [16] only the speed of light under the weak field approximation is required to remain unchanged, and in this paper, without emphasizing weak fields, the speed of light is always thought 1 , so this paper can be seen as an improvement to [16], but the main conclusion remains unchanged.

\section{Proof that the Pressure Inside a Celestial Body Can Appear Infinite as the Coupling Constant of Field Equation Is $-8 \pi G$}

From above discussions, we know that, when the pressure at the boundary of a celestial body is set as zero, the coupling constant is determined as $\gamma=-8 \pi G$. Now, we prove that such coupling constant can lead infinite pressure inside some celestial bodies. I am still going to set $\rho$ as a constant.

The symmetry of the sphere ensures that $p$ is only a function of $r$. From (31), we know $\mathrm{d} p /(\rho+p) \mathrm{d} r=-\mathrm{d} \ln \sqrt{B} /$ $\mathrm{d} r=-\mathrm{d} B / 2 B \mathrm{~d} r$; multiplying both sides by $(\mathrm{d} r / \mathrm{d} \sqrt{D})$, we get

$$
\frac{\mathrm{d} p}{(\rho+p) \mathrm{d} \sqrt{D}}=-\frac{\mathrm{d} B}{2 B \mathrm{~d} \sqrt{D}}
$$

Note that now $\gamma=-8 \pi G$ and considering (28), we get

$$
-\frac{2 \mathrm{~d} p}{(\rho+p) \mathrm{d} \sqrt{D}}=\frac{\mathrm{d} B}{B \mathrm{~d} \sqrt{D}}=-\gamma A_{1}\left(p \sqrt{D}+D^{-1} \int_{0}^{\sqrt{D}} D \rho \mathrm{d} \sqrt{D}\right) \text {. }
$$

Since $\rho$ is a constant, the integral on the right-hand side is easy to calculate, we can get $\{[1 /(p+\rho / 3)]-[1 /(p+\rho)]\}$ $\mathrm{d} p / \mathrm{d} \sqrt{D}=\{\mathrm{d} \ln [1-(8 / 3) \pi G \rho D]\} / 2 \mathrm{~d} \sqrt{D}$, namely, $\{[1 /$ $(p+\rho / 3)]-[1 / p+\rho)]\} \mathrm{d} p=(1 / 2) \mathrm{d} \ln \quad[1-(8 / 3) \pi G \rho D]$; integrating both sides gets $\int_{p}^{0}\{[1 /(p+\rho / 3)]-[1 /(p+\rho)]\}$ $\mathrm{d} p=(1 / 2) \int_{D}^{D_{e}} \mathrm{~d} \ln [1-(8 / 3) \pi G \rho D] ; \quad$ notice that, at 

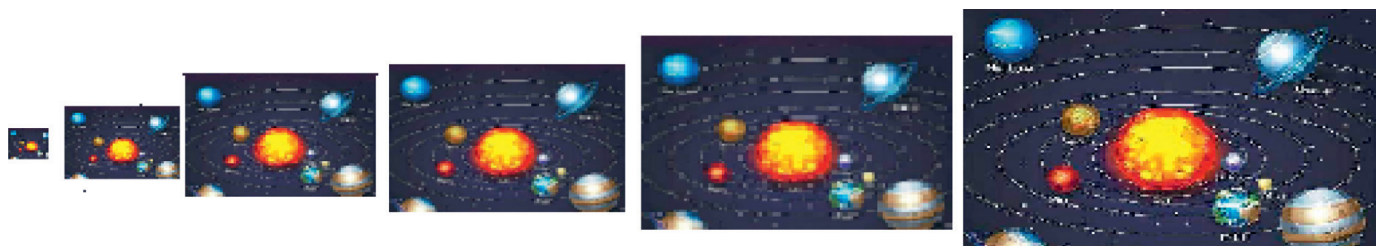

FIgURE 1: Schematic diagram of the Sun and the planets growing at the same time.

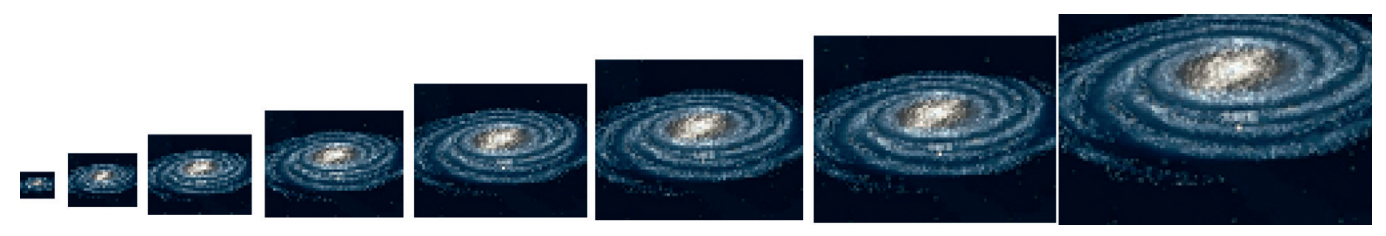

FIGURE 2: Schematic diagram of gradual growth of the Milky Way galaxy.
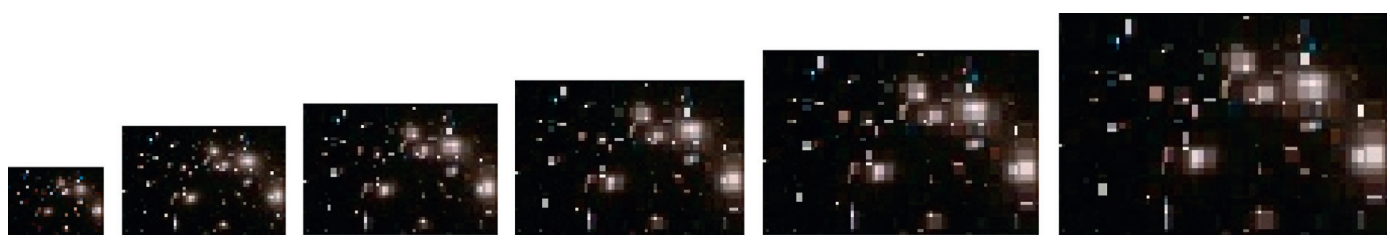

FIgURE 3: Schematic diagram of the generation process of cosmic space.

boundary $p=0$. So, we obtain $p=(\beta \rho-\rho) /(3-\beta)$, where $\beta=\sqrt{(1-8 \pi G \rho D / 3) /\left(1-8 \pi G \rho D_{e} / 3\right)}$. Obviously, for $3=\beta, p=\infty$ and $D=9 D_{e}-(3 / G \rho \pi)$. That is to say, if there are no singularities in a celestial body, it must hold that $9 D_{e}-(3 / G \rho \pi)<0$, which shows that the higher the density is, the smaller the upper bound of the surface's $D$ is. Notice that $D$ is a nonnegative increasing function of $r$, and at the origin, $D=0$. So, the bigger the $\rho$ is, the smaller the radius is, and beyond the critical radius, the internal pressure is going to be infinite. On the other hand, since $\rho=\left(3 M / 4 \pi G r_{e}^{3}\right)$, we have $D=9 D_{e}-(3 / \pi \rho G)=D_{e}\left[9-\left(4 r_{e} / G M\right)\left(r_{e}^{2} / D_{e}\right)\right]$, and taking into account of inverse square forces, $r_{e}^{2} / D_{e}$ does not deviate by much from 1 , so generally say, we have $2 G M / r_{e}<8 / 9$, which is exactly what we did before, in the particular division of $D=r^{2}$.

And, inserting $p=(\beta \rho-\rho) /(3-\beta)$ into (29) gives $B=$ $\left(3 \sqrt{1-8 \pi G \rho D_{e} / 3}-\sqrt{1-8 \pi G \rho D / 3}\right)^{2}\left(1-2 G M / \sqrt{D_{e}}\right) /$ $\left(4-32 \pi G \rho D_{e} / 3\right)$ obviously, for $\beta=3, B=0$, and it can be seen from (3) that the speed of light at this point is zero, such as the speed of light in tangential motion $r \mathrm{~d} \varphi / \mathrm{d} t=\sqrt{B}(r / \sqrt{D})=0$.

So far, we say that the pressure singularity is inseparable from that the speed of light which is zero, and on the other hand, the speed of light is zero is the result of the speed of light which is a variable, which means that the singularity can be avoided as long as the speed of light is constant, which is why this paper emphasizes the constant speed of light.

And again, even though what we have shown here is that the internal pressure becomes singularities when the radius of an object with a constant density increases to a certain extent, for an object with a general density where $\rho$ is a minus function of $r$, that is still true. For example, if the surface density is $\rho^{\prime}$, the radius must not exceed $\sqrt{1 / 3 G \pi \rho^{\prime}}$; otherwise, there will be a singularity.

However, conceptually, for a celestial body of finite density and finite size, its internal pressure should also be finite. The occurrence of a singularity only indicates that our theory is defective or wrong. There is no singularity in nature, and the singularity is only in the mathematical equation. When encountering singularity, people should first think of modifying the theory rather than trying to make the nature meet the needs of the theory, which is the cause of modifying the coupling constant of the field equation in this paper. There is always a danger in the field of astronomy. Because false theories are not easy to be disproved immediately, people like to fantasize about some reasons to prevent these theories from being eliminated immediately. For example, the introduction of dark matter and dark energy is a typical example of this kind of fantasy, which interferes with the correct understanding of nature and hinders the progress of science. Therefore, the identification of the truth and falsehood of the theory should be guided by advanced philosophy in addition to insisting on the connection with reality. Firstly, the outline of the theory should be judged from philosophy. Otherwise, I will get lost in the details of mathematics and walk further and further on the wrong road. We should understand that wrong theories can only explain a phenomenon in isolation and cannot be used to guide practice, especially in other fields. The cycle of the expansion and contraction of the universe fully embodies the idea that things will develop in the opposite direction 
when they become extreme, which is the essence of materialist dialectics, and organically combines the infinity of space-time with the observed fact of the expansion of the universe, so the cycle model of the universe is the only correct one.

\section{Data Availability}

The data used in the paper are available from textbooks. Because this paper is biased towards theoretical description, only a few specific data are used, such as the Hubble constant, the mass and radius of the sun, the precession angle of Mercury, the angle at which light bends on the surface of the sun, and the density of the universe, which can be found in ordinary textbooks, they are reliable.

\section{Conflicts of Interest}

The authors declare that there are no conflicts of interest.

\section{Acknowledgments}

The study was supported by the National Key Research and Development Plan (973 Plan), No. A030101.

\section{References}

[1] L. Lorio, "Gravitational anomalies in the solar system," International Journal of Modern Physics D, vol. 24, no. 6, Article ID 1530015, 2015.

[2] S. Weinberg, Gravitation and Cosmology, Wiley, New York, NY, USA, 2020.

[3] S. Carroll, Lecture Notes on General Relativity, Columbia University, New York, NY, USA, 2013.

[4] L. D. Landau, The Classical Theory of Fields, Pergmon Press, Oxford, UK, 1987.

[5] Einstein, The Meaning of Relativity, 117 pages, Princeton University Press, Princeton, NJ, USA, 1922.

[6] B. C. Tolman, Relativity, Thermodynamics and Cosmology, Oxford Clarendon Press, Oxford, UK, 1934.

[7] J. L. Yang, "Criticism to universal big Bang," Journal of Astrophysics \& Aerospace Technology, vol. 4, no. 1, 2016.

[8] T. F. Felicead, "Theories," Living Reviewing in Relativity, vol. 13, no. 1, 2012.

[9] B. G. W. Abbott, "A three detector observation of gravitational waves from a binary black hole coalescence," Physical Review Letters, vol. 119, pp. 1-16, Article ID 141101, 2017.

[10] B. P. Abbott, "Observation of gravitational wave from a binary black hole merger," Physical Review Letters, vol. 116, 2016.

[11] X. Mei and P. Yu, "Did LIGO really detect gravitational waves?” Journal of Modern Physics, vol. 7, no. 10, pp. 1098-1104, 2016.

[12] C. Mart'inez-Lombilla, L. Trujillo, and H. Johan, "Discovery of disc truncations above the galaies' mid-plane in Milky Way-like galaxies," Monthly Notices of the Royal Astronomical Society, vol. 483, no. 1, pp. 664-691, 2017.

[13] I. Prigogine, J. Geheniau, E. Gunzig, and P. Nardone, "Thermodynamics of cosmological matter creation," Proceedings of the National Academy of Sciences, vol. 85, no. 20, pp. 7428-7432, 2017.

[14] G. R'acz and L. Obos, "Concirdance cosmology without dark energy," Monthly Notices of the Royal Astronomical Society, vol. 469, no. 1, pp. L1-L5, 2017.
[15] P. Bhar and N. Pant, "Relativistic anisotropic stellar models with tolman VII apacetime," Astrophysics and Space Science, vol. 357, 2015.

[16] J. Yang, "Unavoidable correction to the coupling constant in Einstein field equation," International Journal of Advanced Research in Physical Science, vol. 6, no. 11, pp. 4-30, 2019.

[17] Ol ivier S., Blumenthal G. R., Dekel A., et al. APJ, 1990, 35 $610.1086 / 168809$.

[18] J. P. Huchra and J. P. Henry, "A deep abell cluster redshift survey," The Astrophysical Journal, vol. 365, 1990.

[19] M. Yang, "Modification of gravitational field equation and rational solution to cosmological puzzles," Indian Journal of Pharmaceutical Sciences, vol. 5, no. 2, 2010.

[20] J. Gaite, "The fractal geometry of the cosmic web and its formation," Advance in Astronomy, vol. 2019, p. 25, Article ID 6587138, 2019.

[21] S. N. Gurbatov and A. T. Saichev, "Large-scale structure of the universe," Physics-Uspekhi, vol. 55, 2012.

[22] S. L. Blibbikov and A. D. Dolgov, "Cosmological acceleration," Physics-Uspekhi, vol. 62, no. 6, pp. 529-567, 2019.

[23] J. T. Nielsen, A. Guffanti, and S. Sarkar, "Marginal evidence for cosmic acceleration from type ia supernovae," Science Reports, vol. 6, 2016. 